\title{
MCM3: A Novel Proliferation Marker in Oral Squamous Cell Carcinoma
}

\author{
Ludmila de F. Valverde, MSc, ${ }^{*}$ Raiza D. de Freitas, BSc, ${ }^{*}$ Thiago de A. Pereira, PhD, $\dagger$ \\ Marina F. de Resende, MSc, I Ivan M.G. Agra, PhD, $\S$ Jean N. dos Santos, PhD,*\| \\ Mitermayer G. dos Reis, PhD,* Caroline B.S. Sales, PhD,* \\ and Clarissa A. Gurgel Rocha, PhD*\|
}

\begin{abstract}
The present study sought to evaluate and compare the immunoexpression of proteins minichromosome maintenance (MCM) 3 and $\mathrm{Ki}-67$ in oral squamous cell carcinoma (OSCC) to assess the potential of these proteins as markers of cellular proliferation. Twenty-eight cases of OSCC, 9 of tumor-free resection margins (TM), and 4 of non-neoplastic oral mucosa (NNM) were subjected to immunohistochemistry to detect the expression of proteins MCM3 and Ki-67. All OSCCs demonstrated positivity for both proteins. In these tumors, greater MCM3 immunoreactivity was observed in comparison with $\mathrm{Ki}$ 67, whereas TMs and NNMs exhibited greater Ki-67 expression compared with MCM3. The immunoexpression of Ki-67 seemed to be influenced by the inflammatory process, particularly in TM and NNM. Our findings indicate that although both MCM3 and $\mathrm{Ki}-67$ represent reliable markers of cellular proliferation in OSCC, as MCM3 expression does not appear to be influenced by external factors, this protein may emerge as a novel marker of cellular proliferation in these types of tumors.
\end{abstract}

Key Words: squamous cell carcinoma, cellular proliferation, immunohistochemistry

(Appl Immunohistochem Mol Morphol 2018;26:120-125)

$\mathrm{O}$ ral cancer, despite being readily preventable and detectable at early stages, continues to be a public health concern worldwide. ${ }^{1,2}$ The International Agency for

Received for publication November 26, 2015; accepted April 21, 2016. From the *Laboratory of Pathology and Molecular Biology, Oswaldo Cruz Foundation; §Aristides Maltez Hospital; \|Laboratory of Oral Surgical Pathology, School of Dentistry of the Federal University of Bahia; Department of Pathology, School of Medicine of Federal University of Bahia, Bahia; $†$ School of Medicine of the Federal University of Minas Gerais, Minas Gerais; and \$AC Camargo Cancer Center, São Paulo, Brazil.

Supported by National Council for Scientific and Technological Development (CNPq, Brazil). Foundation to Support Research in the State of Bahia (FAPESB, Bahia, Brazil).

The authors declare no conflict of interest.

Reprints: Clarissa A. Gurgel Rocha, PhD, Department of Pathology, School of Medicine of Federal University of Bahia, Av. Araújo Pinho, 62, Salvador, Bahia 40110-150, Brazil (e-mail: cgurgel@ bahia.fiocruz.br).

Copyright (C) 2016 Wolters Kluwer Health, Inc. All rights reserved.
Research on Cancer estimates that approximately 320,000 new cases and 157,000 deaths will occur in 2015 alone, $80 \%$ afflicting developing countries. In Brazil, 15,290 new cases of oral cavity cancer were predicted in 2015, with oral squamous cell carcinoma (OSCC) being the predominant histologic type, as over $95 \%$ of all cases occur at this anatomic site. ${ }^{1,3,4}$ When diagnosed at later stages, oral cancer carries a high morbidity and mortality, and $<50 \%$ of all patients are expected to survive longer than 5 years. ${ }^{1,5}$

The proliferative capacity of neoplastic cells, an important feature in tumor growth ${ }^{6}$ is considered as an important prognostic indicator. ${ }^{7}$ Minichromosome maintenance (MCM) proteins have recently come under investigation, as these helicases play a fundamental role in the replication of eukaryotic DNA by ensuring that this process occurs only once during each cell cycle. ${ }^{7,8}$ The MCM3 protein, similar to other polypeptides comprising the MCM complex, is present at lower intracellular levels in differentiated or quiescent cells. ${ }^{9}$

Classic markers of cellular proliferation, such as protein $\mathrm{Ki}-67$, have been used widely in the assessment of a variety of human tumors, including sarcomas, lymphomas, and breast and prostate neoplasia. ${ }^{10}$ Lee et $\mathrm{al}^{7}$ reported that MCM3 protein expression provides more sensitive and reliable results with which to evaluate the proliferative profile of cell populations, as this protein continues to be expressed for longer than Ki-67. Furthermore, Lameira et $\mathrm{al}^{8}$ emphasized that the protein expression of $\mathrm{Ki}-67$ offers imprecise information with respect to malignant neoplasia, as it incorporates the total fraction of cells within the cell cycle, regardless of whether these cells will eventually undergo differentiation in the absence of any relation to a malignant phenotype. In addition, Ki-67 expression may also occur when DNA synthesis is blocked or in apoptotic cells. ${ }^{8,11}$

The use of MCM proteins as markers of cellular proliferation has been described in some human tumors, such as salivary gland tumors, ${ }^{12}$ thyroid papillary carcinoma, ${ }^{7}$ and melanoma. ${ }^{13}$ In OSCC, MCM2 protein immunoreactivity is considered as a better marker of tumor prognosis ${ }^{14-16}$ than Ki-67. ${ }^{11}$ Nevertheless, to the best of our knowledge, few studies have attempted to assess the role of the protein MCM3 in oral cancer. ${ }^{8,10}$ The present study therefore aimed to evaluate and compare the 
immunoexpression of MCM3 and Ki-67 to assess the potential of these proteins as markers of cellular proliferation in OSCC.

\section{MATERIALS AND METHODS}

The present research proposal received approval from our host institution's review board. The expression of proteins MCM3 and $\mathrm{Ki}-67$ was investigated in 28 cases of OSCC. All tumors were clinically classified by 2 experienced pathologists according to the TNM Classification of Malignant Tumours (2002), in addition to being histologically classified in accordance with the WHO criteria (2005). Nine cases of tumor-free resection margins (TM) were included, with oral epithelial dysplasia (OED) present in $5(55.55 \%)$ cases: $3(60 \%)$ of these were classified as mild, $1(20 \%)$ was moderate, and $1(20 \%)$ as severe. For comparison purposes, the expression of these 2 proteins was also quantified in 4 histologically normal non-neoplastic oral mucosa (NNM) tissue samples obtained from the removal sites of unerupted mandibular third molars in healthy patients - that is, nonsmokers who refrained from alcohol use. Table 1 lists the clinical and histologic characteristics of the OSCC cases studied.

The presence of inflammation was also observed in all OSCC, TM, and NNM samples in accordance with the amount of inflammatory cells dispersed throughout the subjacent connective tissue.

\section{Immunohistochemistry}

Sections (4- $\mu \mathrm{m}$ thick) were obtained from formalinfixed paraffin-embedded specimens. Histologic sections were deparaffinized in xylene, then rehydrated with alcohol, and subsequently subjected to antigen retrieval (MCM3, citrate $\mathrm{pH}$ 9.0; Ki-67, citrate $\mathrm{pH}$ 6.0) under moist heat for 45 minutes to reveal antigenic epitopes. Endogenous peroxidase activity was subsequently blocked (Peroxidase Blocking Solution; Dako, Carpinteria) for 10 minutes under dark conditions, and tissue protein blocking was also performed for 10 minutes (Protein Blocking Solution; Dako). The primary antibodies (MCM3, Clone 101; Dako; Ki-67, Polyclonal; Abcam) were incubated for 18 hours at $4^{\circ} \mathrm{C}$.

After primary antibody incubation, horseradish peroxidase Link and horseradish peroxidase Enzyme reagents (Advance; Dako Corporation) were applied to tissue sections for 20 minutes each. Reactions were developed using 3,3diaminobenzidine (Dako) for 5 minutes in a dark chamber, and all slides were subsequently counterstained with Harris' hematoxylin for 1 minute, and then mounted in natural Canada balsam. For negative controls, each primary antibody was substituted with normal serum of the same isotype.

\section{Immunohistochemical Analysis}

An Aperio digital microscope (Leica Microsystems, Wetzlar, Germany) was used to scan all slides, which were then displayed on an LCD monitor by the Aperio Image Scope software (Leica Microsystems). Five coincident and representative areas $(\times 200$ final amplification $)$ of each OSCC case were selected, with each area containing
TABLE 1. Clinical and Histologic Characteristics of Patients With Oral Squamous Cell Carcinoma

\begin{tabular}{lc}
\hline Clinical Parameters & Total [n $\mathbf{( \% )}]$ \\
\hline Sex & \\
Male & $20(71.42)$ \\
Female & $8(28.58)$ \\
Tumor size & $14(50)$ \\
T1-T2 & $14(50)$ \\
T3-T4 & \\
Metastasis-lymph node & $7(35)$ \\
N0 & $21(75)$ \\
N1-N3 & $23(82.14)$ \\
Metastasis-distance & $5(17.86)$ \\
M0 & \\
Mx & $3(10.72)$ \\
Clinical status & $25(89.28)$ \\
I-II & \\
III-IV & $13(46.42)$ \\
Muscular invasion & $15(53.58)$ \\
Yes & \\
No & $4(14.28)$ \\
Bone invasion & $24(85.72)$ \\
Yes & $2(7.14)$ \\
No & $26(92.86)$ \\
Perineural/vascular invasion & \\
Yes & $19(67.85)$ \\
No & $8(28.58)$ \\
Histologic gradation & $1(3.57)$ \\
Well-differentiated & \\
Moderately differentiated & $15(53.57)$ \\
Poorly differentiated & $8(28.57)$ \\
Anatomic site & $3(10.71)$ \\
Tongue & $2(7.15)$ \\
Floor of mouth & \\
Retromolar region & \\
Gingiva & \\
\hline & \\
&
\end{tabular}

no $<75 \%$ of the tumor parenchyma. The same procedures were likewise carried out with respect to all TM and NNM samples.

\section{Analysis of MCM3 and Ki-67 Proteins}

The nuclear location of each antigen was noted, taking into account the cell type and whether staining occurred in the parenchyma and/or the stroma of OSCC cases, or in the epithelium and/or the lamina propria of TMs and NNMs. In the parenchyma/epithelium, positive and negative cells were first counted in each microscopic field; the number of positive cells was then divided by the total number of cells and semiquantification criteria, as described previously by Gurgel et al, ${ }^{17}$ were applied to obtain scores as follows: negative $(-),<5 \%$ of positively immunostained cells; $1+, 5 \%$ to $25 \% ; 2+, 26 \%$ to $50 \%$; $3+, 51 \%$ to $75 \% ; 4+,>75 \%$ of positively immunostained cells.

\section{Statistical Analysis}

All data were subjected to a statistical analysis, and group comparisons were made under nonparametric testing (Mann-Whitney, Kruskal-Wallis) by the GraphPad Prism Software (GraphPad Software, San Diego). $P$-values corresponding to $\alpha$-that is, $\leq 5 \%$, were considered significant. 


\section{RESULTS}

Low levels of MCM3 and Ki-67 protein expression were observed in well-differentiated tumors, whereas OSCCs that were smaller in size - that is, classified as clinical stages I or II with no evidence of metastatic lymph nodes and no muscle or bone invasion, showed greater immunoexpression for MCM3 (Table 2). In contrast, a higher positivity for the $\mathrm{Ki}-67$ protein was seen in tumors with more aggressive parameters (T3 to T4, metastatic lymph nodes, clinical stages III to IV). Table 2 summarizes the immunoreactivity for MCM3 and Ki-67 with respect to the clinical and histologic parameters pertaining to OSCC.

\section{A Higher MCM3 Expression was Observed in OSCC Compared With TM and NNM}

All cases of OSCC exhibited positivity for the MCM3 protein, which was localized in the nuclei of parenchymal tumor cells. Immunostaining for this protein was predominantly scored as $4+(\mathrm{n}=19 ; 67.85 \%)$, followed by $3+(\mathrm{n}=6 ; 21.42 \%), 2+(\mathrm{n}=2,7.14 \%)$, and $1+(\mathrm{n}=1,3.59 \%)($ Fig. 1). All TMs also demonstrated positivity for MCM3, with staining observed in cells within the epithelial compartment, mostly with a corresponding score of $2+(\mathrm{n}=7 ; 77.77 \%)$, followed by $1+$ $(\mathrm{n}=2 ; 22.23 \%)($ Fig. 1$)$. Among the TM cases with OED $(\mathrm{n}=5)$, all were scored as $2+$. With respect to NNM, staining for MCM3 was more discrete and restricted to the basal layer of the epithelium, with scores of $1+$

TABLE 2. Levels of MCM3 and Ki-67 Protein Immunoexpression in Accordance With the Clinical and Histologic Parameters of OSCC

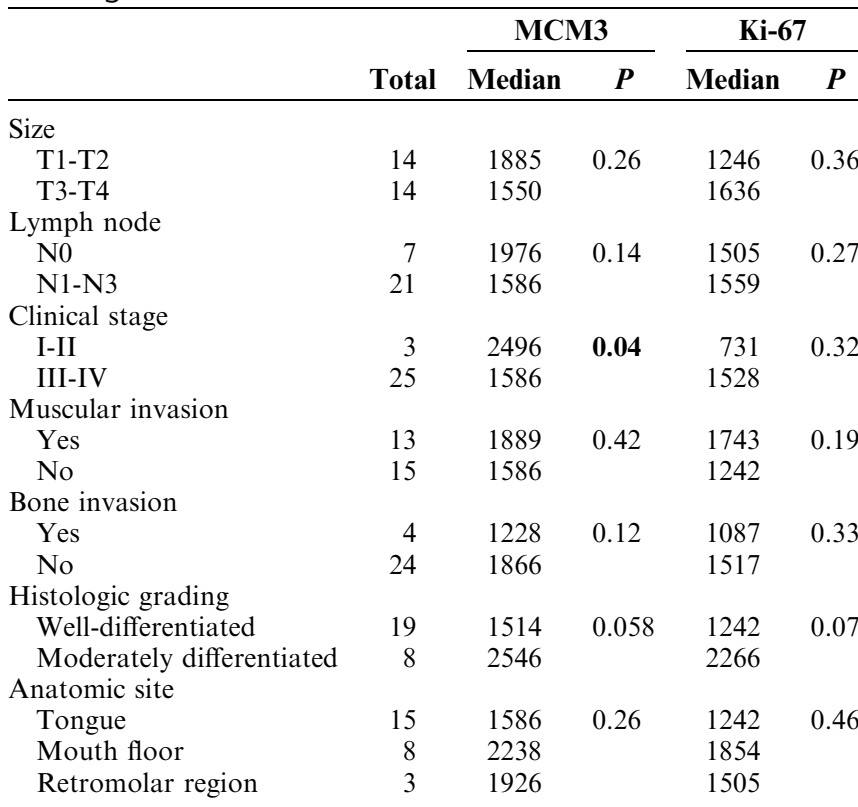

Value in bold are statistically significant $(P<0.05)$.

OSCC indicates oral squamous cell carcinoma; MCM3, minichromosome maintenance 3 .

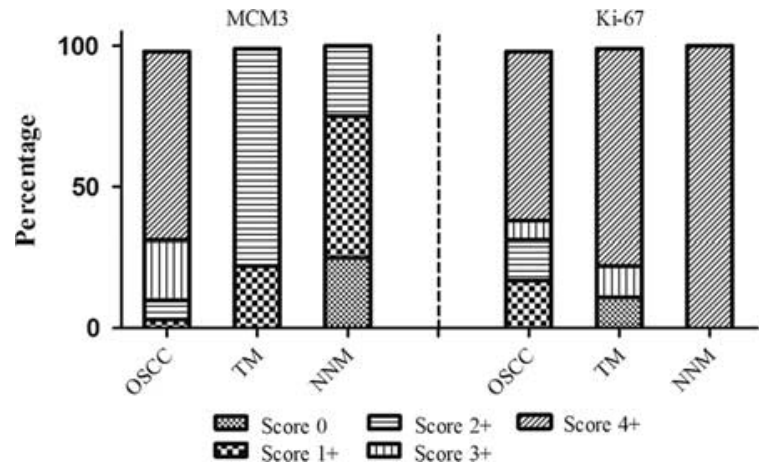

FIGURE 1. Expression distribution of MCM3 and Ki-67 in OSCC, TM, and NNM. MCM3 indicates minichromosome maintenance 3; NNM, non-neoplastic oral mucosa; OSCC, oral squamous cell carcinoma; TM, tumor-free resection margins.

$(\mathrm{n}=2 ; 50 \%), 2+(\mathrm{n}=1,25 \%)$, and $0(\mathrm{n}=1,25 \%)$ (Fig. 1).

Higher MCM3 protein expression was seen in OSCC tumors compared with TMs and NNMs $(P=0.001$; Fig. 2B). Figure 3 illustrates the immunoreactivity for MCM3 in NNM (Figs. 3A, C), TM (Figs. 3E, G), and OSCC (Figs. 3I, K).

\section{Higher Ki-67 Protein Expression in TM and NNM Compared With OSCC}

All samples of OSCC and NNM, in addition to most TMs, demonstrated nuclear immunostaining for protein $\mathrm{Ki}-67$. In OSCC, this expression was seen in both parenchymal and stromal tumor cells. In the first compartment, $4+$ was the predominant score $(\mathrm{n}=17$; $60.71 \%)$, followed by $1+(\mathrm{n}=5 ; 17.85 \%), 2+(\mathrm{n}=4$, $14.28 \%)$, and $3+(\mathrm{n}=2 ; 7.16 \%)$ (Fig. 1). Regarding TMs, 8 cases $(88.88 \%)$ were positive for $\mathrm{Ki}-67$, with staining observed in epithelial cells and the lamina propria. In the epithelium, a score of $4+$ predominated $(\mathrm{n}=7 ; 77.77 \%)$, followed $3+(\mathrm{n}=1,11.11 \%)$ and 0 $(\mathrm{n}=1,11.11 \%)$ (Fig. 1). Among the cases of TM with OED $(\mathrm{n}=5)$, all were scored $4+$. Immunostaining for Ki-67 was scored as $4+$ in all NNM cases (Fig. 1), with positive cells distributed throughout all layers of the epithelium. OSCC tumors exhibited lower Ki-67 expression in comparison with TMs and NNMs $(P=0.03$; Fig. 2A). Figure 3 illustrates the immunoreactivity for Ki-67 in NNM (Figs. 3B, D), TM (Figs. 3F, $\mathrm{H}$ ), and OSCC (Figs. 3J, L).

\section{Proteins MCM3 and Ki-67 Behave Similarly in OSCC, but Differently in TM and NNM}

In OSCC, increased immunoexpression of protein MCM3 was seen in comparison with Ki-67 (Fig. 4). In addition, cells positive for MCM3 were present exclusively in the tumoral parenchyma (Figs. 3I, K), whereas Ki-67 was positively stained in both the parenchymal and the stromal compartments (Figs. 3J, L). Nonetheless, significantly increased immunostaining for Ki-67 was observed in TMs and NNMs compared with 
A

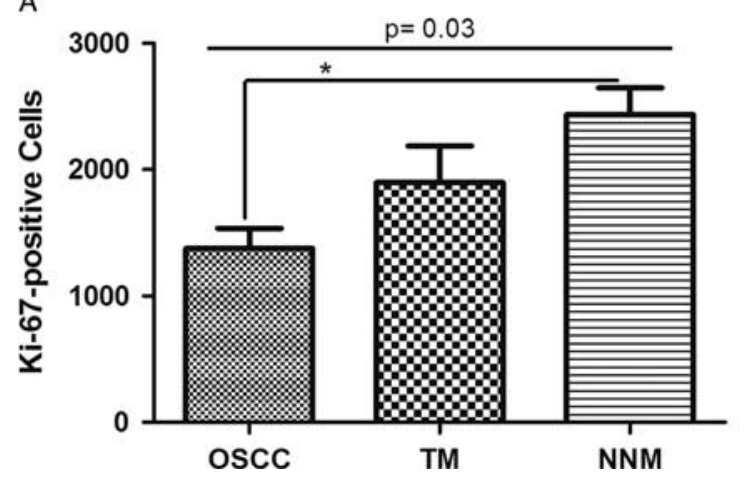

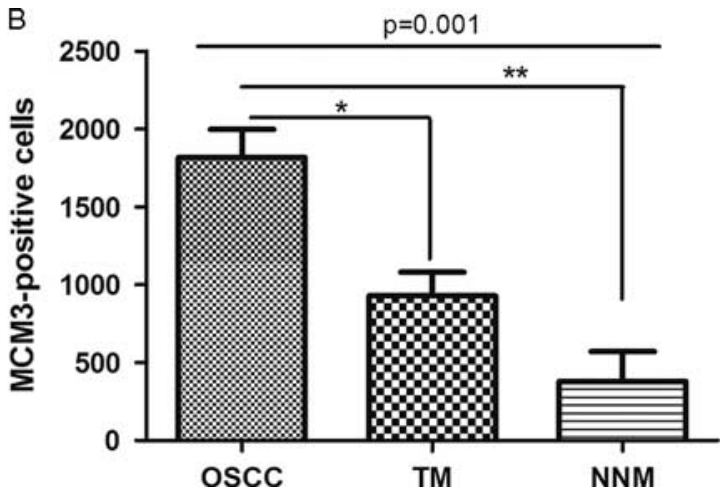

FIGURE 2. Distribution of Ki-67 protein expression (A) and MCM3 protein expression (B) in cases of OSCC, TM, and NNM. MCM3 indicates minichromosome maintenance 3; NNM, non-neoplastic oral mucosa; OSCC, oral squamous cell carcinoma; TM, tumor-free resection margins.

MCM3 ( $P=0.003$ and 0.01 , respectively; Fig. 4). Notably, Ki-67 expression was seen in the epithelium and the lamina propria, whereas MCM3 was localized only in the epithelial compartment (Fig. 3A-H). In addition, in NNM, cells positive for MCM3 were identified in the basal layer of the epithelium, whereas Ki-67-positive cells were distributed throughout all epithelial layers (Fig. 3B).

\section{DISCUSSION}

The present study attempted to assess and compare the expression of 2 proteins associated with cellular proliferation, MCM3 and Ki-67, in OSCC, TM, and NNM. In OSCC samples, greater immunostaining for MCM3 was observed in comparison with $\mathrm{Ki}-67$. In contrast, TMs and NNMs had higher Ki-67 expression in comparison with MCM3. Interestingly, particularly in TMs and NNMs, the immunoreactivity for Ki-67 seemed to be influenced by inflammation, as evidenced by the greater proliferative activity in epithelial cells.

The Ki-67 protein is one of the most studied markers of cellular proliferation. Its expression is initiated in the $\mathrm{S}$ phase, ${ }^{17,18}$ increases throughout the $\mathrm{G} 2$ phase progressively, peaks in G1 phase, ${ }^{18}$ and then disappears rapidly when cells reach G0. ${ }^{9}$ The fraction of Ki-67-positive cells is frequently associated with the clinical course of tumors, such as cancers of the breast ${ }^{19}$ and the lung. ${ }^{20}$ Studies have shown that tumors with elevated Ki-67 expression are associated with an increased risk of recurrence and lower survival rates. ${ }^{21-23}$ Relatedly, in OSCC, Ki-67 has been described as an important predictive marker of recurrence ${ }^{24}$ and survival. $^{25}$
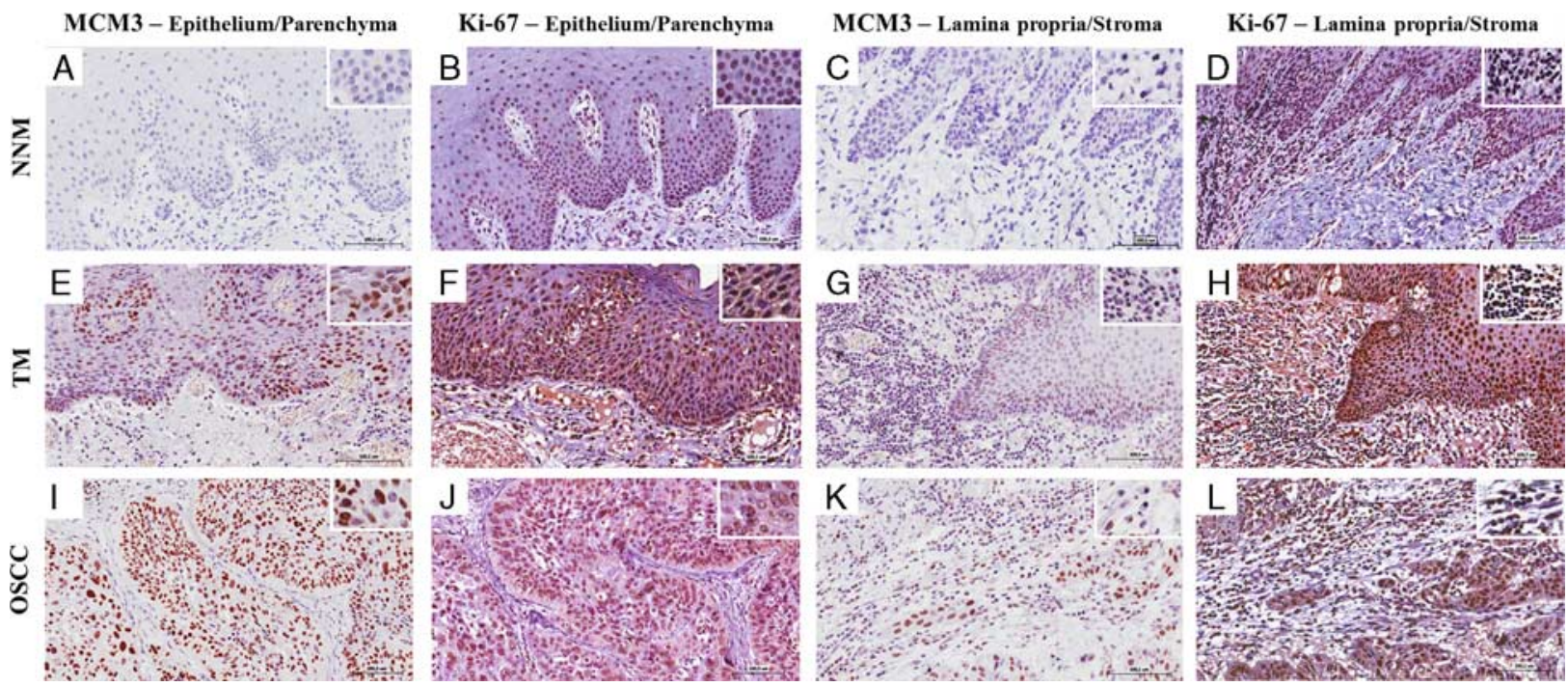

FIGURE 3. Immunostaining for minichromosome maintenance (MCM) 3 and Ki-67 in cases of non-neoplastic oral mucosa (NNM), tumor margin (TM), and oral squamous cell carcinoma (OSCC). MCM3: A and C, negatively scored NNM. E and G, TM exhibiting nuclear staining localized only in the epithelial compartment. I and K, OSCC exhibiting cells positive for MCM3 exclusively in the tumoral parenchyma. Ki-67: B, Positively scored epithelial cells distributed throughout all epithelial layers in NNM. D, Positively scored inflammatory cells distributed in the lamina propria of NNM. F and H, Ki-67 expression in the epithelium and the lamina propria of TM. J and L, positive cells in both the parenchymal and the stromal compartments. 


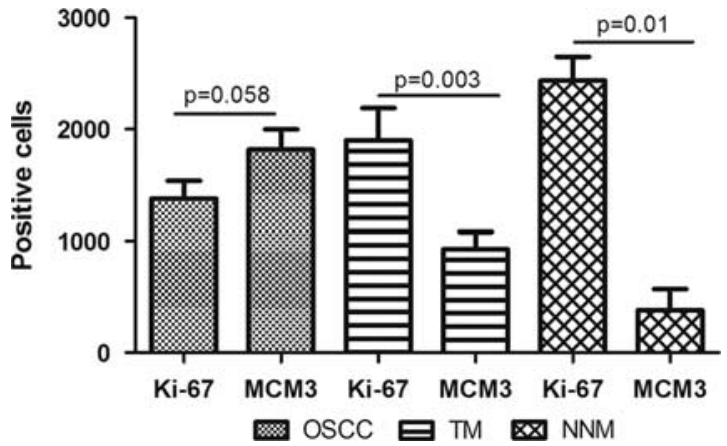

FIGURE 4. Comparison of $\mathrm{Ki}-67$ and $\mathrm{MCM} 3$ protein expression between OSCC, TM, and NNM. MCM3 indicates minichromosome maintenance 3; NNM, non-neoplastic oral mucosa; OSCC, oral squamous cell carcinoma; TM, tumor-free resection margins.

Unfortunately, it has not yet been possible to establish a threshold level for Ki-67 detection that would allow for the differentiation of tumors with varying prognoses. ${ }^{26,27}$ In breast cancer, for example, a previous study suggested a level of $25 \%$ for $\mathrm{Ki}-67$-positive cells, although prospective validation studies have not yet been conducted. ${ }^{27}$

The 6 members of the MCM protein family (MCM2-7) comprise a group of DNA-binding proteins that play an important role in the initiation and regulation of DNA replication and cell cycle progression. ${ }^{7,12,28}$ Notably, the expression of MCM3, a helicase fundamental to eukaryotic DNA replication, ${ }^{7,8,15}$ is maintained for longer than that of $\mathrm{Ki}-67,{ }^{7}$ continuing until the transition between phases G0 and G1. ${ }^{15}$

Simon and Schwacha ${ }^{29}$ concluded that because the MCM protein complex plays a role in cellular proliferation, any alterations that lead to an increased activity by this helicase are thus associated with cancer development. To this end, studies have indeed reported that increased MCM3 expression is associated with a worse prognosis in gliomas, ${ }^{30}$ salivary gland tumors, ${ }^{12}$ thyroid carcinoma, ${ }^{7}$ and melanoma. ${ }^{13}$ In OSCC and premalignant oral lesions, MCM3 is regarded as a prognostic and diagnostic marker ${ }^{10}$ that some consider to be superior to $\mathrm{Ki}-67 .{ }^{8}$

One of our findings was that less aggressive OSCCs of smaller size, without metastatic lymph nodes or any muscle or bone invasion and classified as clinical stages I or II, exhibited more marked immunostaining for MCM3 and $\mathrm{Ki}-67$. This evidence must be interpreted in the context of the tumor biology, because in early stages, the proliferative tumor compartment is predominantly responsible for the death of malignant cells, whereas at later stages, neoplastic cells acquire an invasive capability and promote tumor progression. ${ }^{6}$ Furthermore, more differentiated tumors exhibit lower MCM3 and Ki-67 immunoreactivity, which is generally associated with an improved prognosis, ${ }^{14}$ as these cells still retain some differentiation potential.

Particularly in comparison with NNM, OSCCs exhibit higher MCM3 protein expression, indicating that cellular proliferation is a key event in tumor growth and progression. ${ }^{6,7}$ In the NNM samples considered herein, MCM3-positive cells were seen only in the basal layer of the epithelium, whereas the Ki-67 protein was found to be distributed throughout all layers of the epithelium. Moreover, Ki-67 expression was higher in NNMs than in OSCCs. Considering that inflammation of the oral mucosa is a frequent occurrence, taken together with the presence of Ki-67-positive cells dispersed throughout the connective tissue (lamina propria/stroma), it is logical to conclude that the inflammatory process seems to influence the expression of Ki-67, ${ }^{31-33}$ but not MCM3. Within this context, Ki-67 expression has been previously associated with the secretion of proinflammatory factors, such as $\mathrm{NF}-\kappa \mathrm{B}$ in chronic inflammatory dermatosis-for example, psoriasis vulgaris. ${ }^{34}$ Furthermore, some studies have demonstrated that the secretion of cytokines and growth factors during inflammatory events may consequently modulate cellular proliferation, growth, and differentiation. ${ }^{31-33}$ Relatedly, a previous report found a mitogenic effect resulting from PDGF growth factor expression in keratinocyte cell cultures, with consequently increased levels of Ki-67. ${ }^{35}$

Premalignant lesions of the mouth, which often precede OSCC, are generally difficult to differentiate from benign alterations. As such, the use of biomarkers of cellular proliferation can aid in prognosis establishment, as greater numbers of cells undergoing division increases the chances of genomic instability, thereby promoting the emergence of aggressive clones. ${ }^{36}$ Accordingly, some studies attempting to evaluate the use of biomarkers capable of detecting early-stage oral dysplasia ${ }^{16,37}$ propound the superiority of MCM family proteins over Ki67. Corroborating these reports, our results demonstrate a more reliable pattern of immunostaining for MCM3 in TMs, despite the higher levels of $\mathrm{Ki}-67$ protein immunoreactivity detected. Nevertheless, we must stress that the expression of this protein indeed appears to be influenced by the inflammatory process in both NNMs and TMs.

It is clear that biomarkers of cellular proliferation can aid in the interpretation of morphologic alterations occurring during neoplastic initiation and transformation processes. The analysis of proliferative tumor activity, in association with clinical and histopathological data, provides useful information regarding the biological and prognostic behavior of neoplasia - for example, by providing an estimation of the rate of tumor growth as well as assisting in treatment planning. ${ }^{29}$ Despite widespread investigation on the use of biomarkers to improve the assessment of oral cancer prognosis, the standardized clinical application of prognostic markers remains a goal yet to be achieved..$^{15}$

In conclusion, the search for alternative markers of cellular proliferation must continue, particularly in light of the potential they offer to significantly enhance the clinical management of patients affected by malignant neoplasia, as well as in the hope that these will eventually lead to reduced morbidity and mortality not only in epithelial 
dysplasia, but also in other forms of cancer. Our findings suggest that although both MCM3 and Ki-67 proteins represent proven markers of cell proliferation in oral cancer, the expression of MCM3 does not appear to be influenced by external factors - for example, inflammation, which seems to be the case when quantifying Ki-67 immunoexpression. Nevertheless, further studies must be conducted to assess the influence (or a lack thereof) that proteins MCM3 and Ki-67 may have on the inflammatory process. It is our hope that the present results regarding MCM3 immunoreactivity in OSCCs and TMs may serve to further the emergence of MCM3 as a novel marker of cellular proliferation in these types of tumors.

\section{ACKNOWLEDGMENTS}

The authors thank Andris K. Walter for providing English translation and consulting services.

\section{REFERENCES}

1. Rodrigues PC, Miguel MC, Bagordakis E, et al. Clinicopathological prognostic factors of oral tongue squamous cell carcinoma: a retrospective study of 202 cases. Int J Oral Maxillofac Surg. 2014; 43:795-801.

2. Honorato J, Rebelo MS, Dias FL, et al. Gender differences in prognostic factors for oral cancer. Int J Oral Maxillofac Surg. 2015; 44:1205-1211.

3. Bhargava A, Saigal S, Chalishazar M. Histopathological grading systems in oral squamous cell carcinoma: a review. J Int Oral Health. 2010;2:1-10.

4. Jemal A, Bray F, Center MM, et al. Global cancer statistics. $C A$ Cancer J Clin. 2011;61:69-90.

5. Adrien J, Bertolus C, Gambotti L, et al. Why are head and neck squamous cell carcinoma diagnosed so late? Influence of health care disparities and socio-economic factors. Oral Oncol. 2014;50:90-97.

6. Hanahan D, Weinberg RA. Hallmarks of cancer: the next generation. Cell. 2011;144:646-674.

7. Lee YS, Ha SA, Kim HJ, et al. Minichromosome maintenance protein 3 is a candidate proliferation marker in papillary thyroid carcinoma. Exp Mol Pathol. 2010;88:138-142.

8. Lameira AG, Pontes FS, Guimarães DM, et al. MCM3 could be a better marker than Ki-67 for evaluation of dysplastic oral lesions: an immunohistochemical study. J Oral Pathol Med. 2014;43:427-434.

9. Endl E, Kausch I, Baack M, et al. The expression of Ki-67, MCM3, and p27 defines distinct subsets of proliferating, resting, and differentiated cells. $J$ Pathol. 2001;195:457-462.

10. Rezvani G, Andisheh-Tadbir A, Ashraf MJ, et al. Evaluation of minichromosome maintenance-3 (MCM3) in oral squamous cell carcinoma. J Dent (Shiraz). 2015;16:87-92.

11. Szelachowska J, Dziegiel P, Jelen-Krzeszewska J, et al. MCM-2 protein expression predicts prognosis better than Ki-67 antigen in oral cavity squamocellular carcinoma. Anticancer Res. 2006;26(3B): 2473-2478.

12. Ashkavandi ZJ, Najvani AD, Tadbir AA, et al. MCM3 as a novel diagnostic marker in benign and malignant salivary gland tumors. Asian Pac J Cancer Prev. 2013;14:3479-3482.

13. Nodin B, Fridberg M, Jonsson L, et al. High MCM3 expression is an independent biomarker of poor prognosis and correlates with reduced RBM3 expression in a prospective cohort of malignant melanoma. Diagn Pathol. 2012;7:82-92.

14. Kodani I, Osaki M, Shomori K, et al. Minichromosome maintenance 2 expression is correlated with mode of invasion and prognosis in oral squamous cell carcinomas. J Oral Pathol Med. 2003;32:468-474.

15. Gueiros LA, Coletta RD, Kowalski LP, et al. Clinicopathological features and proliferation markers in tongue squamous cell carcinomas. Int J Oral Maxillofac Surg. 2011;40:510-515.
16. Scott IS, Odell E, Chatrath $P$, et al. A minimally invasive immunocytochemical approach to early detection of oral squamous cell carcinoma and dysplasia. Br J Cancer. 2006;94:1170-1175.

17. Gurgel CA, Ramos EA, Azevedo RA, et al. Expression of Ki-67, p53 and p63 proteins in keratocyst odontogenic tumours: an immunohistochemical study. $J$ Mol Histol. 2008;39:311-316.

18. Gerdes J, Li L, Schlueter C, et al. Immunobiochemical and molecular biologic characterization of the cell proliferation-associated nuclear antigen that is defined by monoclonal antibody Ki-67. Am J Pathol. 1991;138:867-873.

19. Scholzen T, Gerdes J. The Ki-67 protein: from the known and the unknown. J Cell Physiol. 2000;182:311-322.

20. Kim CH, Lee HS, Park JH, et al. Prognostic role of p53 and Ki-67 immunohistochemical expression in patients with surgically resected lung adenocarcinoma: a retrospective study. $J$ Thorac Dis. 2015;7: 822-833.

21. de Azambuja E, Cardoso F, de Castro G Jr, et al. Ki-67 as prognostic marker in early breast cancer: a meta-analysis of published studies involving 12,155 patients. Br J Cancer. 2007;96: 1504-1513.

22. Nishimura R, Osako T, Okumura $\mathrm{Y}$, et al. Ki-67 as a prognostic marker according to breast cancer subtype and a predictor of recurrence time in primary breast cancer. Exp Ther Med. 2010;1: $747-754$.

23. Tashima R, Nishimura R, Osako T, et al. Evaluation of an optimal cut-off point for the Ki-67 index as a prognostic factor in primary breast cancer: a retrospective study. PLoS One. 2015;10:e 0119565.

24. Wangsa D, Ryott M, Avall-Lundqvist E, et al. Ki-67 expression predicts locoregional recurrence in stage I oral tongue carcinoma. $\mathrm{Br}$ $J$ Cancer. 2008;99:1121-1128.

25. Wang Z, Zhang B, Jiang L, et al. RACK1, an excellent predictor for poor clinical outcome in oral squamous carcinoma, similar to Ki67. Eur J Cancer. 2009;45:490-496.

26. Polley MY, Leung SC, McShane LM, et al. An international Ki67 reproducibility study. J Natl Cancer Inst. 2013;105:1897-1906.

27. Petrelli F, Viale G, Cabiddu M, et al. Prognostic value of different cut-off levels of Ki-67 in breast cancer: a systematic review and meta-analysis of 64,196 patients. Breast Cancer Res Treat. 2015;153: 477-491.

28. Forsburg SL. Eukaryotic MCM proteins: beyond replication initiation. Microbiol Mol Biol Ver. 2004;68:109-131.

29. Simon NE, Schwacha A. The MCM2-7 replicative helicase: a promising chemotherapeutic target. Biomed Res Int. 2014;2014: 549719-549733.

30. Hua C, Zhao G, Li Y, et al. Minichromosome maintenance (MCM) family as potential diagnostic and prognostic tumor markers for human gliomas. BMC Cancer. 2014;14:526-534.

31. de Paula AM, Carvalhais JN, Domingues MG, et al. Cell proliferation markers in the odontogenic keratocyst: effect of inflammation. J Oral Pathol Med. 2000;29:477-482.

32. Kaplan I, Hirshberg A. The correlation between epithelial cell proliferation and inflammation in odontogenic keratocyst. Oral Oncol. 2004;40:985-991.

33. Birajdar SS, Radhika M, Paremala K, et al. Expression of Ki-67 in normal oral epithelium, leukoplakic oral epithelium and oral squamous cell carcinoma. J Oral Maxillofac Pathol. 2014;18: $169-176$.

34. Doger FK, Dikicioglu E, Ergin F, et al. Nature of cell kinetics in psoriatic epidermis. J Cutan Pathol. 2007;34:257-263.

35. Rollman O, Jensen UB, Ostman A, et al. Platelet derived growth factor (PDGF) responsive epidermis formed from human keratinocytes transduced with the PDGF beta receptor gene. $J$ Invest Dermatol. 2003;120:742-749.

36. González-Moles MA, Plaza-Campillo J, Ruiz-Ávila I, et al. Asymmetrical proliferative pattern loss during malignant transformation of the oral mucosa. J Oral Pathol Med. 2014;43:507-513.

37. Gouvêa AF, Vargas PA, Coletta RD, et al. Clinicopathological features and immunohistochemical expression of p53, Ki-67, MCM2 and MCM-5 in proliferative verrucous leukoplakia. J Oral Pathol Med. 2010;39:447-452. 